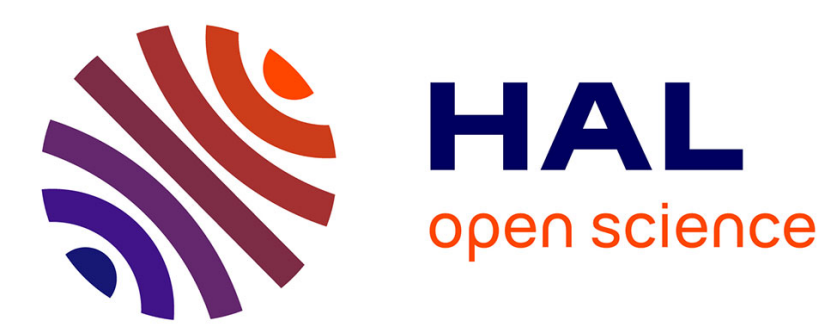

\title{
Entropy in halide perovskites
}

Claudine Katan, Aditya D Mohite, Jacky Even

\section{To cite this version:}

Claudine Katan, Aditya D Mohite, Jacky Even. Entropy in halide perovskites. Nature Materials, 2018, 17 (5), pp.377 - 379. 10.1038/s41563-018-0070-0 . hal-01774679

\section{HAL Id: hal-01774679 \\ https://hal.science/hal-01774679}

Submitted on 26 Sep 2018

HAL is a multi-disciplinary open access archive for the deposit and dissemination of scientific research documents, whether they are published or not. The documents may come from teaching and research institutions in France or abroad, or from public or private research centers.
L'archive ouverte pluridisciplinaire HAL, est destinée au dépôt et à la diffusion de documents scientifiques de niveau recherche, publiés ou non, émanant des établissements d'enseignement et de recherche français ou étrangers, des laboratoires publics ou privés. 


\section{Entropy in halide perovskites}

\section{Claudine Katan, Aditya D. Mohite and Jacky Even discuss the possible impact of various entropy contributions (stochastic structural fluctuations, anharmonicity and lattice softness) on the optoelectronic properties of halide perovskite materials and devices.}

Halide perovskites have undeniably remarkable characteristics, which are interesting for next-generation technologies. Yet, moderate carrier diffusion lengths and mobilities are hard to reconcile with other experimental observations pointing towards long carrier lifetimes, low carrier effective masses or short Urbach tails in the absorption spectrum. Moreover, exciton screening at room temperature, hot carrier effects and the relaxation of carriers toward the band edges, are other important microscopic processes that are yet poorly understood. Providing a clear picture of the behaviour of these materials has proven challenging, also due to the fact that all the above-mentioned optoelectronic properties are affected by the unusually strong structural fluctuations undergone by halide perovskites.

In classic semiconductors, structural fluctuations are usually represented by a thermal bath of phonons, an ensemble of quasiparticles each representing a quantized vibration at a specific frequency. In these systems atomic displacements away from equilibrium are small, thus the vibrational density of states is described within the harmonic approximation, with the energy potential function showing a single minimum around the equilibrium position (Fig. 1a, blue line). Small departures from this ideal behaviour, known as weak anharmonicity (Fig.1a, red line), result in phonon-phonon collisions that lead to temperature-dependent phonon linewidths and non-zero thermal conductivity. 
In contrast, charge carriers moving in hybrid perovskite compounds find a highly perturbed electrostatic landscape, due to stochastic (random) structural fluctuations of the organic and inorganic components and, more broadly, to all the sources of lattice softness ${ }^{1}$. These phenomena and their impact on the optoelectronic properties of halide perovskites have received little scrutiny. Stochastic structural fluctuations have two origins: (i) the energy potential of the halide-perovskite lattice shows a double-well, strongly anharmonic shape (Fig.1a, green line, and Fig.1b), leading to disordered motion of the halides perpendicular to the metal-metal axis; ${ }^{2,3}$ (ii) the charge anisotropy of the organic cations (for instance methylammonium, $\mathrm{MA}^{+}$or formamidinium $\mathrm{FA}^{+}$) lead them to move in a disordered tumbling fashion within the cuboctahedral lattice cavities (Fig. 1c). Frozen at low temperature, these organic dipoles keep disordered orientations, a glassy behaviour that has been predicted theoretically ${ }^{1}$ and observed through dielectric and thermodynamic experiments. ${ }^{4}$ These unconventional features are reminiscent of atomic disorder observed in two other well-known classes of materials: plastic crystals ${ }^{5}$ and oxide perovskites. ${ }^{6}$

At a basic level, the direct impact of stochastic cation reorientations on the carrier transport processes can be gauged in the framework of the so-called dipolar scattering mechanism. This specific microscopic mechanism, rarely considered for classical semiconductors, provides a crude description of carrier scattering by uncorrelated random dipolar fluctuations (Fig. 1b,c). ${ }^{7}$ Its direct influence on carrier transport can be evaluated by computing the dipolar scattering relaxation time $\left(\tau_{\mathrm{d}}\right)$ in the effective mass $(\mathrm{m})$ approximation, $\tau_{d}\left(E / k_{B} T\right)=\frac{2 \pi \epsilon \hbar^{2} \sqrt{2 k_{B} T}}{P^{2} e^{2} N_{p} \sqrt{m}\left(\cos \left(\theta_{q P}\right)\right)^{2}} \frac{\sqrt{E / k_{B} T}}{g\left(E / k_{B} T\right)}$, where $E$ is the carrier energy, $g$ a function describing the screening of the electrostatic interaction, $\theta_{q P}$ the angle between the fluctuating electric dipole 
$\boldsymbol{P}$ and vector $\boldsymbol{q}$ defining the elastic change in wavevector (Fig. 1c), $N_{P}$ the dipole concentration, $\hbar$ is the reduced Planck constant, $k_{B}$ is Boltzmann constant, $T$ is temperature, $\varepsilon$ is the dielectric constant and $e$ is the electric charge. ${ }^{8}$

The carrier mobility is then obtained by numerically integrating over the thermal distribution of carriers. For low carrier concentrations (such as $10^{14} \mathrm{~cm}^{-3}$ in the weak screening regime) a computed maximum mobility as high as $\sim 900 \mathrm{~cm}^{2} \mathrm{~V}^{-1} \mathrm{~s}^{-1}$ for $\mathrm{MAPbI}_{3}$ (considering $\mathrm{m}=0.21, \varepsilon=6$ and $\mathrm{P}=2.85 \mathrm{D}$ ) can be reached by taking into account $\mathrm{MA}^{+}$disordered reorientations. This value is significantly higher than the mobilities experimentally measured in this material, suggesting that the dipolar scattering of charge carriers induced by the organic cations is unlikely the limiting factor in MA-based perovskites — and will similarly have no role in $\mathrm{FAPbI}_{3}$ or $\mathrm{CsPbI}_{3}$, whose cations have small or vanishing electric dipole.

The most remarkable differences between halide perovskites and classic semiconductors are related to the phonon bath itself, mostly related to the fluctuations of the halide perovskite lattice. This exhibits a very low stiffness ${ }^{9}$ and large anharmonicity, ${ }^{2}$ and more generally the phonon vibrational density of states lies at a lower energy, thus promoting electron-phonon collision processes (Fig. 1b and Figs.1d-e). In such cases, perturbative approaches break down and more complex theoretical strategies, such as the stochastic self-consistent harmonic approximation, must be considered. For example, in $\mathrm{CsSnI}_{3}$ perovskite, a proper treatment of phonon-phonon interactions using such method revealed huge modifications of the vibrational density of states and in turn sizeable renormalization of the electronic band gap. ${ }^{2}$ Yet, insights into these effects on monoelectronic states close to the band gap and more generally on the optoelectronic properties of halide perovskites deserve further theoretical and experimental investigations. ${ }^{10}$ Alternatively, a more basic understanding of the impact on carrier mobility of 
large ionic displacements can be gained from the dipolar scattering model by considering the dipole induced by the motion of the halide atoms (Fig. 1b). For instance, an iodine displacement of $0.4 \AA^{3}$ leads to a significant decrease in the maximum allowed carrier mobility $\left(\sim 160 \mathrm{~cm}^{2} \mathrm{~V}^{-1} \mathrm{~s}^{-}\right.$ ${ }^{1}$ ); this suggests that dipolar fluctuations due to the anharmonicity of the halides motion have a stronger impact on charges behavior with respect to those due to the organic cations. Yet it is important to mention that the latters may also have an additional indirect impact: indeed, in hybrid perovskites molecular rotations couple with the lattice strain renormalizing the elastic constants, as evidenced by optical spectroscopy, ${ }^{9}$ and can thus possibly affect the halides motion.

The understanding of the effects of lattice softness can also benefit from earlier work on oxide perovskites, where order-disorder effects coexist with displacive dynamics, ${ }^{6}$ and lowfrequency polar optical modes, corresponding to the above-mentioned halide vibrations (Fig. 1b), strongly interact through nonlinear local coupling with acoustic modes (Fig.1d,e). Organic and inorganic halide perovskites undergo similar coupling mechanisms. ${ }^{1,9}$ Additional investigations are needed to better monitor the importance of the acoustic phonon anharmonicity and of the extreme lattice softness on carrier scattering processes. The presence of unusual phonon bottlenecks may be another consequence of sizeable anharmonicity and low lying vibrational density of states of the perovskite lattice. ${ }^{11}$ Its impact on hot carrier effects and on carrier mobilities requires further elucidation.

The same holds for the strong coupling regime associated to non-polar interactions between local distortions and charge carriers, which leads to the formation of small polarons. ${ }^{12}$ This phenomenon may lead to the formation of two types of charge carriers having different lifetimes, effective masses and other characteristics. Organic cations seem to favour formation of 
small polarons. They may also contribute to the ultralow heat conductivities of hybrid perovskites. ${ }^{11}$

It is actually the study of heat conduction, as well as of other macroscopic material properties depending on low-energy relaxation processes and the vibrational density of states such as specific heat, mechanical stiffness or dielectric susceptibility - , that can improve the microscopic understanding of halide perovskites and provide guidance to optimize their use in optoelectronic devices. Among the recent experimental attempts along this path, one may mention detailed studies on the light-induced emergence and activation of molecular rotation, electron-phonon interactions and polaron formation. ${ }^{13-15}$ The building-up of such interactions in the context of operating devices shall provide further insight on metastability and nonequilibrium phenomena in perovskites. 


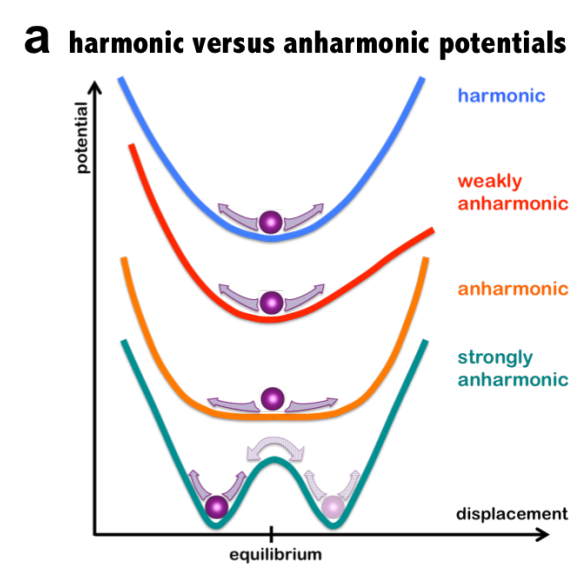

d

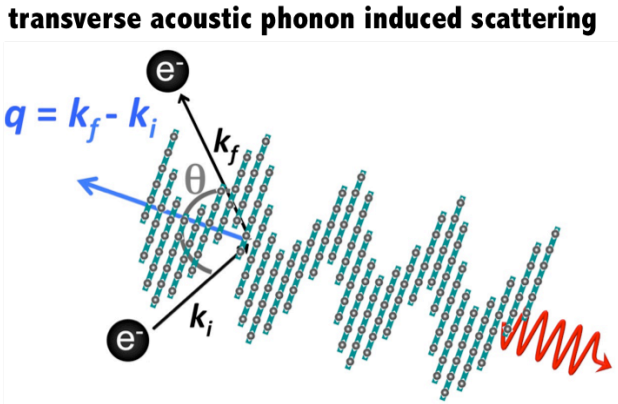

b anharmonic motion induced dipolar scattering

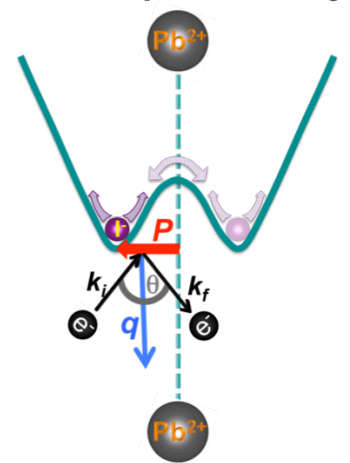

e longitudinal acoustic phonon induced scattering

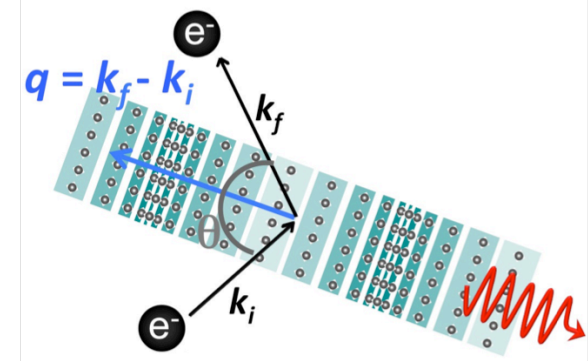

Fig. 1. Sources of dynamic disorder in halide perovskites. a, Various energy potentials shapes, from harmonic to strongly anharmonic. The violet sphere represents an atom moving in the potential around its equilibrium position. b-e, Scattering events leading a charge (electron or hole, black sphere) to change its wavevector from $\boldsymbol{k}_{\boldsymbol{i}}$ to $\boldsymbol{k}_{\boldsymbol{f}}$ due to the interaction with: $\mathbf{b}$, the anharmonic motion of a halide atom (in the example shown, the violet sphere represents an iodine atom, the dashed line is a guide to the eye to locate the equilibrium position on the axis connecting the two lead atoms); $\mathbf{c}$, the stochastic motion of the organic cation (a $\mathrm{MA}^{+}$cation is shown in the example, with carbon, nitrogen and hydrogen atoms shown as grey, light-blue and green spheres, respectively); d, transverse and e, longitudinal acoustic phonon modes, respectively represented as shear and compression waves moving the perovskite lattice planes (green-shaded regions with grey spheres representing atoms positions). $\boldsymbol{P}$ is the electric dipole, $\boldsymbol{q}=\boldsymbol{k}_{f} \boldsymbol{k}_{i}$ the elastic change in wavevector, $\theta_{\mathrm{qP}}$ the angle between vectors $\boldsymbol{P}$ and $\boldsymbol{q}, \theta$ the angle between $\boldsymbol{k}_{f}$ and $\boldsymbol{k}_{i}$. 
Acknowledgments: The work at Los Alamos National Laboratory (LANL) was supported by LDRD program (A.D.M). Part of the work was conducted at the Center for Integrated Nanotechnologies (CINT), a U.S. Department of Energy, Office of Science user facility. The work in France was supported by Agence Nationale pour la Recherche (TRANSHYPERO project).

Claudine Katan is at Univ Rennes, ENSCR, INSA Rennes, CNRS, ISCR (Institut des Sciences Chimiques de Rennes) - UMR 6226, F-35000 Rennes, France; Aditya D. Mohite is at the Los Alamos National Laboratory, Los Alamos, New Mexico 87545, USA; Jacky Even is at Univ Rennes, INSA Rennes, CNRS, Institut FOTON - UMR 6082, F-35000 Rennes, France. e-mail: claudine.katan@univ-rennes1.fr,jacky.even@insa-rennes.fr

\section{References}

1. Even, J., Carignano, M. \& Katan, C. Molecular disorder and translation/rotation coupling in the plastic crystal phase of hybrid perovskites. Nanoscale 8, 6222-6236 (2016).

2. Patrick, C. E., Jacobsen, K. W. \& Thygesen, K. S. Anharmonic stabilization and band gap renormalization in the perovskite CsSnl 3. Phys. Rev. B 92, (2015).

3. Marronnier, A. et al. Structural Instabilities Related to Highly Anharmonic Phonons in Halide Perovskites. J. Phys. Chem. Lett. 8, 2659-2665 (2017).

4. Fabini, D. H. et al. Dielectric and Thermodynamic Signatures of Low-Temperature Glassy Dynamics in the Hybrid Perovskites $\mathrm{CH}_{3} \mathrm{NH}_{3} \mathrm{Pbl}_{3}$ and $\mathrm{HC}\left(\mathrm{NH}_{2}\right)_{2} \mathrm{Pbl}_{3}$. J. Phys. Chem. Lett. 376-381 (2016).

5. Lynden-Bell, R. M. \& Michel, K. H. Translation-rotation coupling, phase transitions, and elastic phenomena in orientationally disordered crystals. Rev. Mod. Phys. 66, 721-762 (1994).

6. Bussmann-Holder, A., Büttner, H. \& Bishop, A. R. Polar-Soft-Mode-Driven Structural Phase Transition in SrTiO 3. Phys. Rev. Lett. 99, (2007). 
7. Boardman, A. D. The theory of dipole scattering in semiconductors. Proc. Phys. Soc. 85, 141-148 (1965).

8. Senanayak, S. P. et al. Understanding charge transport in lead iodide perovskite thin-film field-effect transistors. Sci. Adv. 3, e1601935 (2017).

9. Létoublon, A. et al. Elastic Constants, Optical Phonons, and Molecular Relaxations in the High Temperature Plastic Phase of the $\mathrm{CH}_{3} \mathrm{NH}_{3} \mathrm{PbBr}_{3}$ Hybrid Perovskite. J. Phys. Chem. Lett. 7, 37763784 (2016).

10. Giustino, F. Electron-phonon interactions from first principles. Rev. Mod. Phys. 89, (2017).

11. Yang, J. et al. Acoustic-optical phonon up-conversion and hot-phonon bottleneck in lead-halide perovskites. Nat. Commun. 8, 14120 (2017).

12. Neukirch, A. J. et al. Polaron Stabilization by Cooperative Lattice Distortion and Cation Rotations in Hybrid Perovskite Materials. Nano Lett. 16, 3809-3816 (2016).

13. Wu, X. et al. Light-induced picosecond rotational disordering of the inorganic sublattice in hybrid perovskites. Sci. Adv. 3, e1602388 (2017).

14. Ghosh, T., Aharon, S., Etgar, L. \& Ruhman, S. Free Carrier Emergence and Onset of Electron-Phonon Coupling in Methylammonium Lead Halide Perovskite Films. J. Am. Chem. Soc. 139, 18262-18270 (2017).

15. Taylor, V. C. A. et al. Investigating the Role of the Organic Cation in Formamidinium Lead lodide Perovskite Using Ultrafast Spectroscopy. J. Phys. Chem. Lett. 895-901 (2018). 